\title{
List of chapters
}

\section{Part I - Persistence through time}

Chapter 1, Introduction \& definitions

p. 19

Chapter 2, Problems with presentism

p. 27

Chapter 3, The presentist perdurantist view

p. 35

Chapter 4, The problem of change in temporary intrinsic properties p. 45

Chapter 5, Coincidence and vagueness ……………………………... p. 67

Chapter 6, The ship of Theseus .................................................... p. 85

Chapter 7, The worm view and the stage view................................. p. 91

Chapter 8, Four-dimensionalism and common sense ........................ p. 103

Chapter 9, The modal objection ....................................................... p. 113

\section{Part II - Persistence across possible worlds}

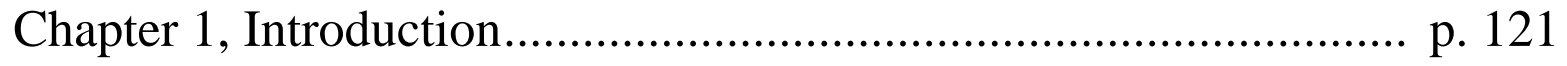

Chapter 2, Modal realism ............................................................. p. 125

Chapter 3, Straightforward trans-world identity ………………….... p. 129

Chapter 4, Modal counterpart theory ............................................... p. 133

Chapter 5, Partial trans-world identity ……………………………... p. 137

Chapter 6, Modal perdurants .......................................................... p. 153

Chapter 7, Genuine actualism - modal fictionalism.......................... p. 175

Chapter 8, Abstractionism-Ersatzism-Actualism .............................. p. 197

Chapter 9, Abstractionism and trans-world identity ……………..... p. 207

Chapter 10, Abstractionism and counterpart theory ……………….. p. 217

Chapter 11, Abstractionism and modal perdurants............................ p. 233

Chapter 12, Bundle-bundle-bundle theory ………………………..... p. 241

List of figures ....................................................................... p. 269

Bibliography …………………………………………………... p. 271 
